\title{
VISCOELASTIC PROPERTIES OF CAT TENDON: EFFECTS OF TIME AFTER DEATH AND PRESERVATION BY FREEZING*+
}

\author{
LARRY S. MATTHEWS $\ddagger$ and DUNALD ELLIS $\ddagger$
}

Section of Orthopaedic Surgery and the

Department of Physical Medicine and Rehabilitation, University of Michigan Medical Center. Ann Arbor, Michigan 48104. U.S.A.

\begin{abstract}
In this study postmortem changes in the character of the stress-strain limit cycle were analyzed for cat extensor communis and extensor lateralis tendons maintained in a stream of Ringer's solution and loaded with a short series of symmetrical triangular-wave stress cycles. The mechanical behavior of these tendons was compared with that of similar tendons which had been preserved by freezing.

It was found that the overall stiffness (average apparent elastic modulus) of these tendons did not change significantly in the first 3 hrs after death and that the shape characteristics of the stress-strain curves changed little under these conditions.

Tendons preserved by freezing displayed stress-strain limit cycles similar to those for fresh material. The average moduli for frozen tendons were lower than those from fresh tendons to a degree which was statistically significant.
\end{abstract}

\section{INTRODUCTION}

IN RECENT years there has been increasing interest in the mechanical properties of fibrous connective tissue. The correction of deformities involving connective tissue abnormalities and the design of devices for protection of the body against damaging stresses are both influenced by information about the mechanical properties of this tissue.

Much future work on the mechanics of connective tissue will undoubtedly involve specimens from amputation or necropsy. It is difficult to obtain this material immediately after amputation or death, and often it would be convenient to freeze specimens for testing at a later date.

The purpose of this study is to determine whether the visco-elastic properties of tendon change shortly after death and whether they are altered by storing tendon in the frozen state.
Previous studies have usually approached this problem indirectly or not at all. Some investigators, for example Cronkite (1936) and Walker et al. (1964), have used only material which had been embalmed or preserved by freezing. Smith (1954), however. found that rabbit cruciate ligaments become less extensible (elastic modulus increases) and react elastically to greater loads (elastic limit increases) within $1 \mathrm{hr}$ after death. He suggested that results of experiments on fresh ligament might be valid only if the experiment was performed within thirty minutes after death. Nunley (1958), in studies of canine ligamentum flavum noted an increase in the strength of this tissue associated with storage in the frozen state. Cronkite (1936) found no difference between tensile strengths of embalmed tendons and fresh tendons examined 24-48 hrs after death. Van Brocklin and Ellis (1965) noted no difference between

\footnotetext{
*Received 27 December 1967.

†This study conducted as part of the University of Michigan Orthetics Research Project which receives partial support through funds granted by the Vocational Rehabilitation Administration.

$\ddagger 1045$ East Ann Street. Ann Arbor. Michigan 48104. U.S.A.
} 
the viscoelastic character of fresh human toe extensor tendons examined on the day of the amputation and specimens which had been frozen.

Two studies comparable to ours were carried out by Viidik, Sandquist and Magi (1965) and by Viidik and Lewin (1966). In the first, load-elongation curves for 62 rabbit anterior cruciate ligaments were evaluated with respect to elastic modulus, failure energy, elongation at rupture, and rupture sites. For these specimens, stored moist at room temperature from $0-96 \mathrm{hrs}$, they found no change in the test parameters with time after death. In the more recent study Viidik and Lewin (1966) examined the same parameters in ligaments which had been stored in saline. frozen, and tanned. No change in elastic modulus was associated with storage in saline at $20^{\circ} \mathrm{C}$ for five hours or with storage in the frozen state. Twenty-four hrs storage in saline at $4^{\circ} \mathrm{C}$, however, was associated with a decrease in elastic modulus. For every method of storage evaluated, however, these investigators found significant change in at least one of the parameters. They concluded that none of the examined methods of storage was satisfactory for the preservation of the mechanical characteristics of fresh connective tissue.

In our study we have evaluated changes in apparent elastic modulus, changes in length, and variability in shape of the stress-strain curves as related to time after death and to storage in the frozen state.

\section{MATERIALS AND METHODS}

\section{Specimens}

Extensor communis and extensor lateralis tendons from eight large adult cats were examined. Each cat was sacrificed using intraperitoneal sodium pentobaritol, and its tendons immediately removed. One tendon from each cat was tested immediately, while the remainder were frozen at $-10^{\circ} \mathrm{C}$.

\section{Apparatus:}

A low-capacity, dead-weight testing machine, as previously described by Van Brocklin and Ellis (1965), was used in these experiments. Specimens were positioned in the apparatus between a pair of wedge-jaw clamps. Tension on the specimen was varied by adding or removing water from a bucket at a predetermined flow rate. This tension was continuously measured by means of a standard proving ring instrumented with strain gauges. Specimen strain was measured by a strain gauge clip attached to the specimen between the clamps.

Signal changes were amplified and recorded on a Grass Polygraph as stress (force per unit area) and strain (change in length per unit initial length) as functions of time. Stress was plotted as a function of strain by means of a Mosely Autograf $x-y$ recorder.

\section{Procedure}

A single fresh specimen taken from each of the eight cats immediately after death was weighed and measured. Cross-sectional area was calculated using $1 \cdot 13$ as the average specific gravity for fresh cat tendon. The stress gain on the instrumentation was adjusted for cross-sectional area, and the specimen was placed in the apparatus. Tests were performed initially from 5 to 17 mins after the tendons were removed from the animal. They were repeated at 60 mins, 120 mins and 180 mins after removal from the animal. The specimens were maintained in the apparatus in a flowing stream of Ringer's solution at $25-27^{\circ} \mathrm{C}$. They were not removed from the apparatus between tests.

Each test consisted of a series of four or seven cycles of stress increasing and de-

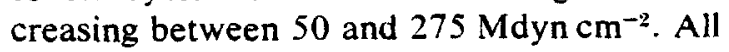
stress cycles for a given tendon were identical and had equal loading and unloading rates. Tendons loaded at stress rates greater than

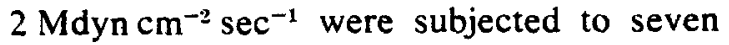
stress cycles in each series. Those loaded

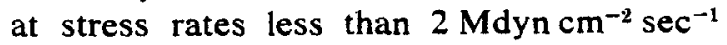
were subjected to four cycles in each series, in order to give comparable test periods. Be- 
tween periods of testing a stress of $50 \mathrm{Mdyn}$ $\mathrm{cm}^{-2}$ was maintained on the specimen.

The eight fresh specimens were grouped as four pairs. Pairs were stressed at loading and unloading rates of approximately $1,3,10$ and

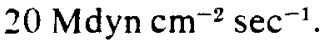

Throughout the period of examination of a fresh tendon it was possible to document any changes in length with time.

Forty frozen specimens (maintained at $-10^{\circ} \mathrm{C}$ for approximately 2 weeks) were tested through one series of cycles. The stress application rate for each group of frozen specimens from a single animal was adjusted to approximate that at which the corresponding fresh tendon had been examined.

The systems for measurement of stress and strain were calibrated at the beginning and end of this set of experiments and were found to have retained their initial calibration to within an error of less than 3 per cent of their outputs for each calibration point.

\section{Quantitation of data}

In order to characterize quantitatively the stress-strain cycle for each test we employed three numerical parameters. These were the average apparent elastic modulus (tangent modulus) for the stress-strain limit cycle. average modulus, and the r.m.s. deviations of the apparent elastic modulus from the average for the loading and for the unloading half cycles. modulus r.m.s. deviations. The average modulus provides a measure of overall stiffness of the tendon during the test. The modulus r.m.s. deviations provide measures of the nonlinearity of the stress-strain relations for the loading and unloading half cycles. For each test we determined values for these parameters on the basis of the slope of the stress-strain curves measured at stress levels of $80,120.160,200$ and $240{\mathrm{Mdyn} \mathrm{cm}^{-2}}^{-2}$ for the loading and for the unloading half cycles.

Since the tendons preserved by freezing were not the same tendons that were tested while fresh, a direct comparison of the average modulus for fresh and frozen material on an individual tendon basis was not feasible. To evaluate the difference between the average moduli of fresh and frozen specimens. we used a ' $t$ ' test for significance in the difference between the mean of the average moduli for all frozen tendons and the mean of the average moduli for all initial tests on fresh tendons.

\section{RESULTS}

For each fresh tendon tested, the form of the stress-strain limit cycle remained nearly unchanged throughout the series of four tests performed. This is suggested by Fig. 1 which shows the stress-strain plot obtained for a fresh tendon for which the test cycle loading

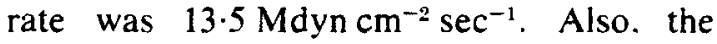
form of the stress-strain limit cycle for tendons preserved by freezing was similar to that for fresh tendons.

However, fresh specimens were observed to shorten by a fraction of a percent during the interval between tests (relative displacement to the left of the traces in Fig. 1).

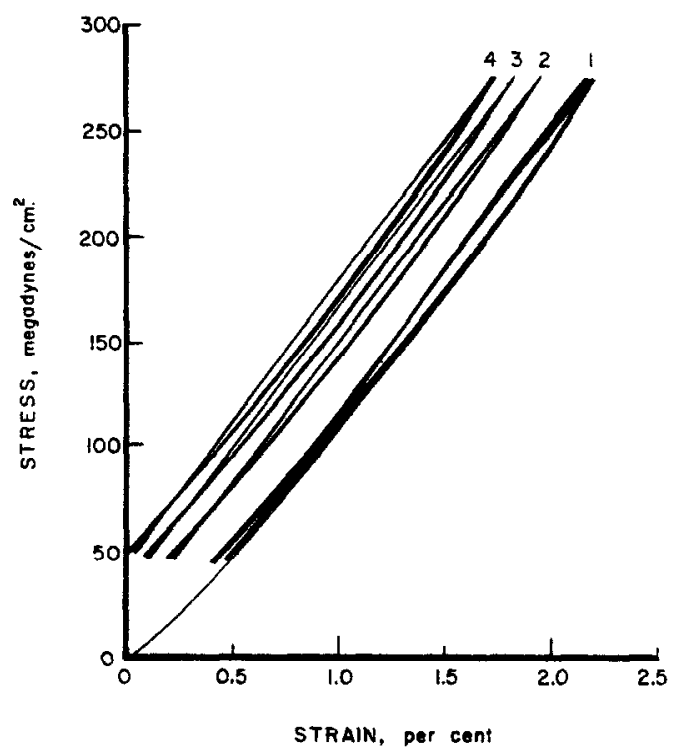

Fig. 1. Stress-strain reiations recorded for cat extensor communis tendon subjected to four serjes of seven triangular wave stress cycles each, with stress application and removal rates of $13.5 \mathrm{Mdyn} \mathrm{cm}^{-2} \mathrm{sec}^{-1}$. beginning at (1) 5 mins, (2) 60 mins, (3) 120 mins, and (4) 180 mins, after death. 


\section{Overall stiffness}

In Fig. 2 the average modulus is plotted as a function of the time interval between removal of the tendon from the cat and the start of each test for each of the eight fresh tendons. These curves show some fluctuation in average modulus for a tendon from one test to another, but fail to indicate any large or systematic change in the average modulus in the $3 \mathrm{hr}$ interval after death.

A ' $t$ ' test for significance in the difference between the means of the average elastic

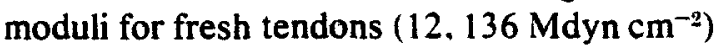
and that for frozen tendons $(10,893 \mathrm{Mdyn}$ $\mathrm{cm}^{-2}$ ) indicated that this difference was significant at the 99 per cent confidence level.

\section{Nonlinearity}

The modulus r.m.s. deviation for the loading half cycle for all tests is plotted against stress application rate in Fig. 3. Here the nonlinearity of the loading half of the limit cycle is seen to have been comparable for fresh and frozen specimens and not to have

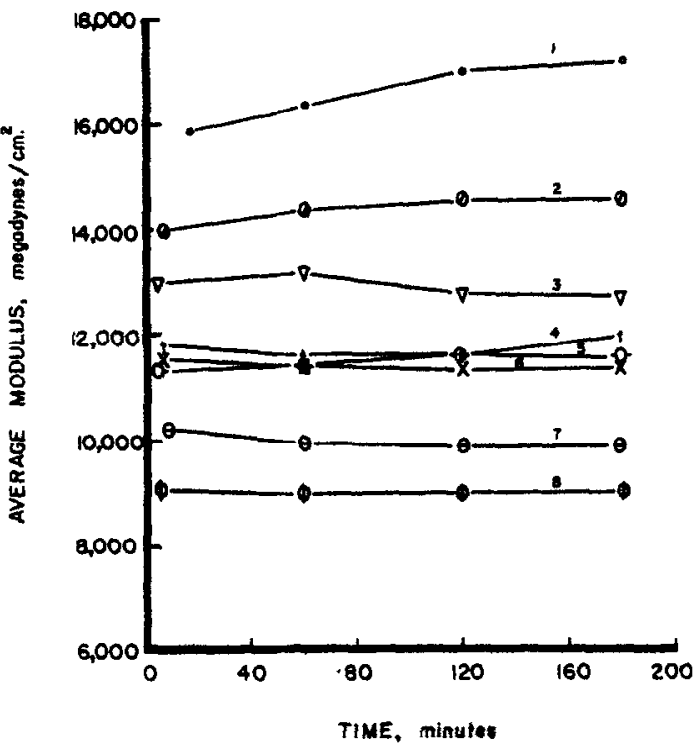

Fig. 2. Average apparent elastic modulus for the stressstrain limit cycle, as a function of time between death and the beginning of the stress cycle series, for each of eight cat extensor communis tendons tested in the fresh condition. Cycle stress application and removal rates are: (1) $25 \cdot 3$, (2) $9 \cdot 0$, (3) $13 \cdot 5$, (4) $1 \cdot 28$, (j) $3 \cdot 22$, (6) $23 \cdot 5$, (7) $1 \cdot 37$ and (8) $3 \cdot 2 \mathrm{Mdyn}^{-2} \mathrm{sec}^{-1}$

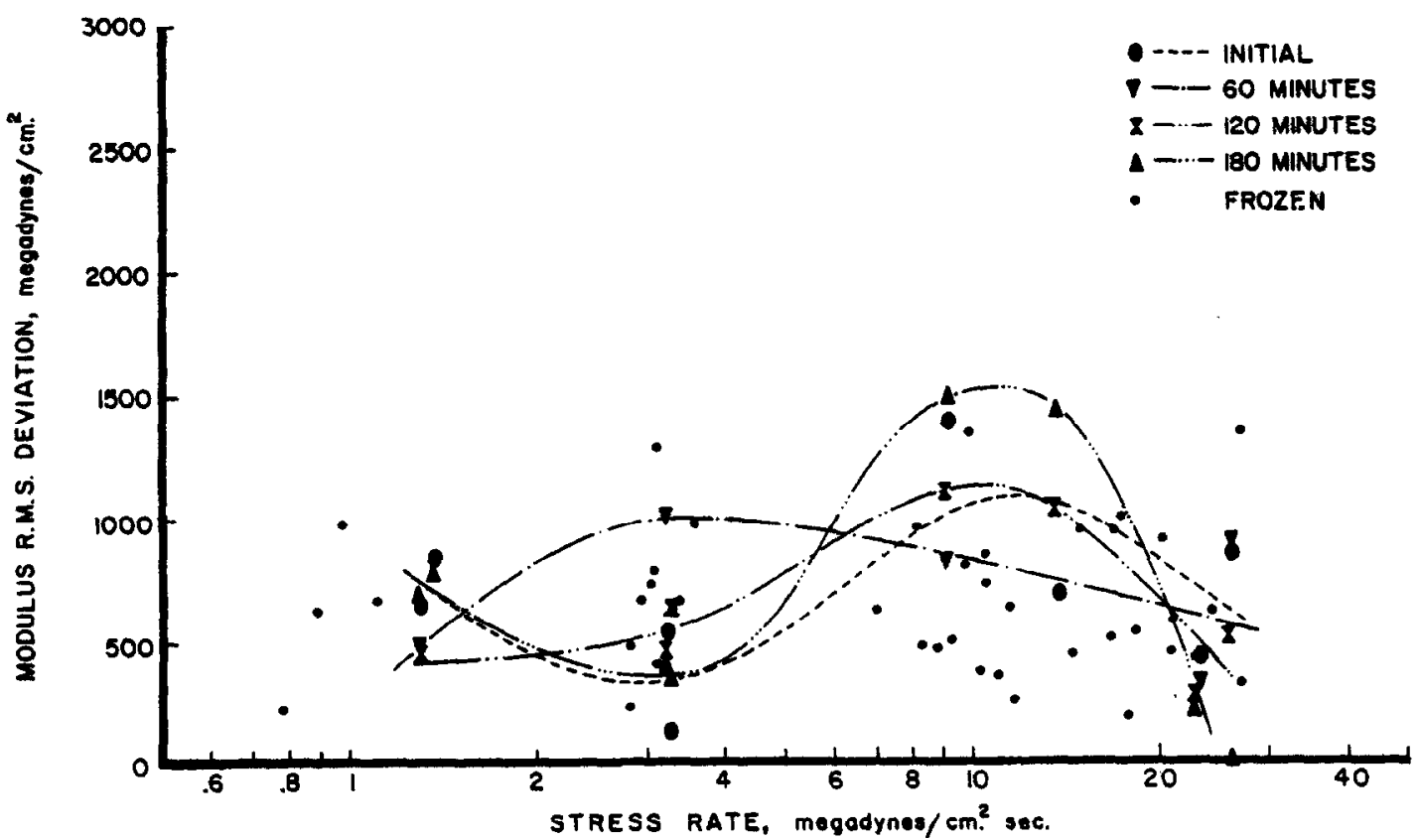

Fig. 3. Modulus r.m.s. deviation for the loading half of the stress-strain limit cycle, as a function of stress application rate, for cat extensor communis and extensor laterlis tendons. 
changed systematically during the $3 \mathrm{hr}$ period covered by the tests.

Figure 4 presents a similar plot for the unloading half cycle. Except for stress rates of approximately $3 \mathrm{Mdyn} \mathrm{cm}^{-2} \mathrm{sec}^{-1}$ at which frozen specimens tended to show more nonlinearity. the nonlinearity of the stressstrain relation is seen to have been similar for fresh and frozen tendons for the unloading half cycle. Again little or no systematic change seems to have occurred with time.

A comparison of Figs. 3 and 4 indicates the stress-strain relation to have been more nonlinear for the unloading half cycle than for the loadin? half cycle.

\section{Shortening}

In order to compare the inter-test shortening observed in the series of tests on fresh tendon, we assumed shortening to have begun when the tendon was excised and to have progressed at a constant rate for the first 60 mins. With this assumption. the zero from which shortening was measured was deter- mined by extrapolation from the time of the start of the first test, using a shortening rate equal to the average shortening rate in the first inter-test interval. Figure 5 shows a plot of shortening. relative to this derived zero. for each fresh tendon tested. as a function of time. The curve labeled ' 0 ' in this figure was obtained for a fresh tendon which

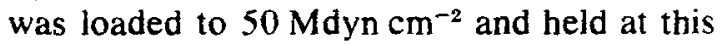
stress for 3 hrs without cycling through a test series. Since this tendon showed initial viscoelastic lengthening which was absent in other tendons due to their stress history, the 'starting' time from which the zero was extrapolated was taken at the time when the tendon had maximum length (approximately 20 mins after stress was applied). These curves indicate that shortening tended to continue throughout the $3 \mathrm{hr}$ period and that its rate tended to decrease with time. However, considerable variation among tendons was noted. This variation appears to have depended somewhat on the stress rate during the test.

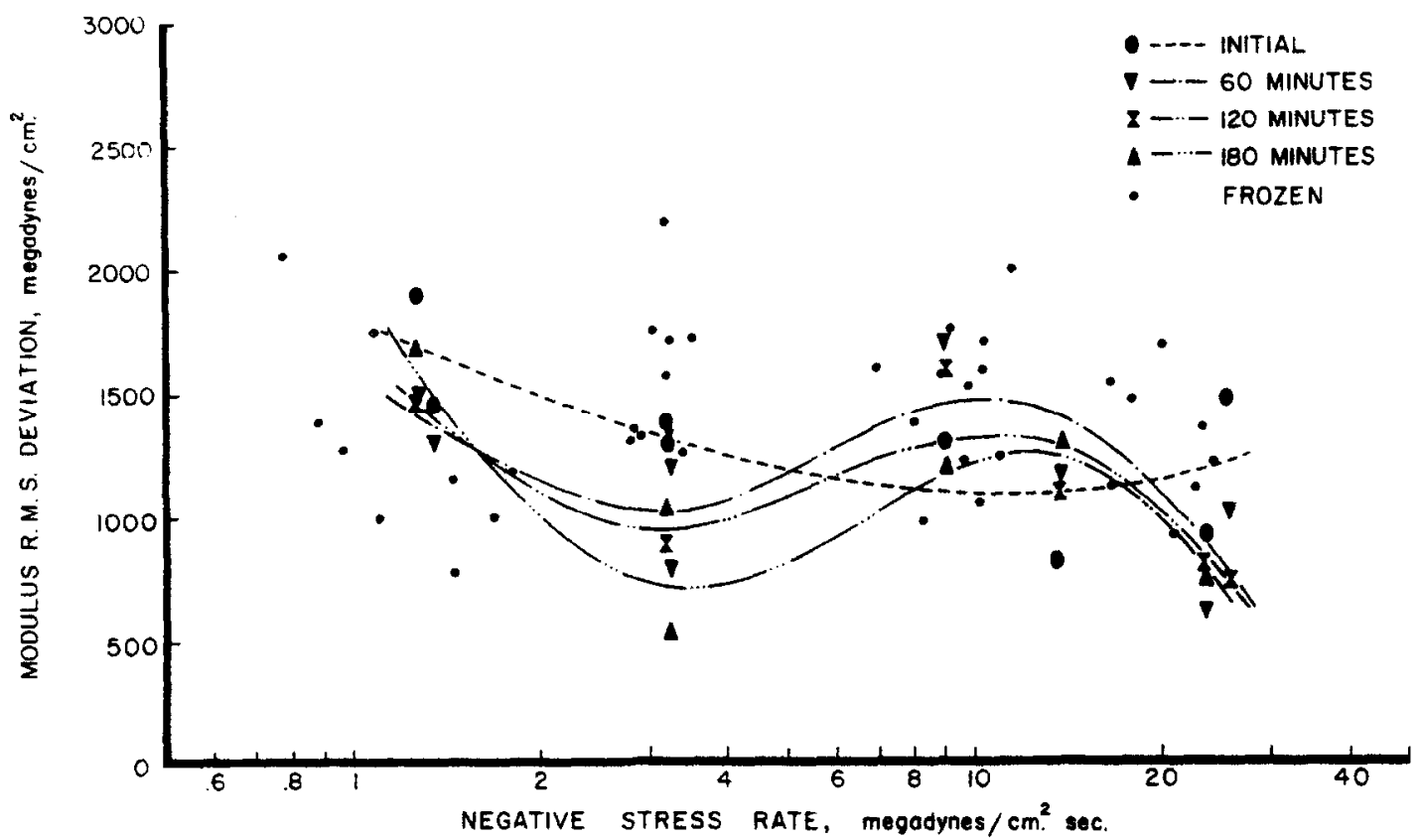

Fig. 4. Modulus r.m.s. deviation for the unloading half of the stress-strain limit cycle. as a function of stress application rate, for cal extensor communis and extensor lateralis tendons. 


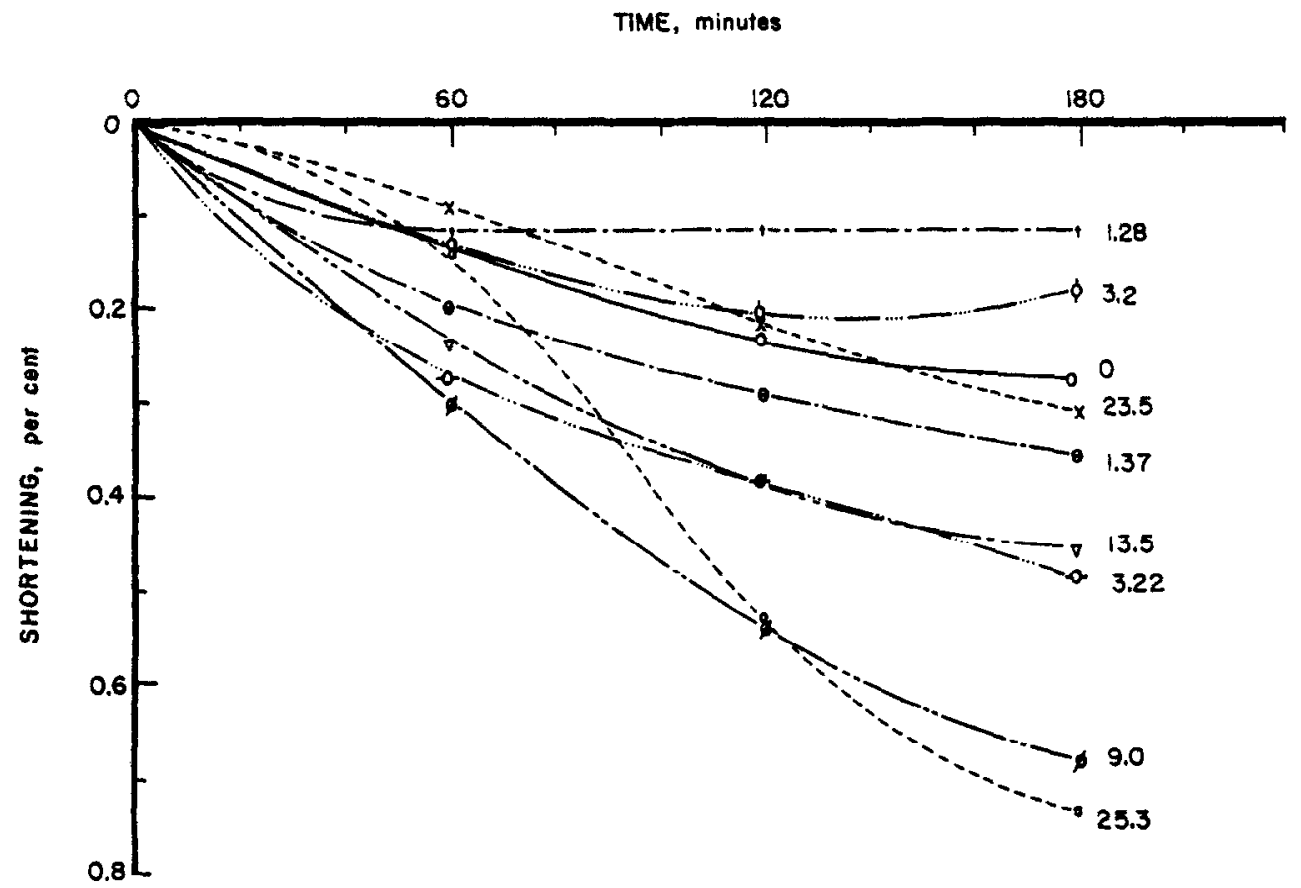

Fig. 5. Cumulative inter-test shortening at a stress of $50 \mathrm{Mdyn}^{-2}$, relative to extrapolated 'initial' length, for nine fresh cat extensor communis tendons, as a function of time after death. The number by which a curve is identified is the rate of stress application and removal during the test, in Mdyn $\mathrm{cm}^{-2} \mathrm{sec}^{-1}$.

In addition to the fresh tendons for which shortening was examined, one frozen tendon was subjected to a three-test series at test stress rates of $11.0 \mathrm{Mdyn} \mathrm{cm}^{-2} \mathrm{sec}^{-1}$ with inter-test intervals of $1 \mathrm{hr}$. This tendon showed 0.18 per cent shortening in the first inter-test interval, but no measurable shortening in the second inter-test interval or after the third test. This shortening was considerably less than that observed in fresh tendons subjected to a comparable stress history but was within the range of shortening behavior recorded for fresh tendons.

\section{Discussion}

The experiments performed demonstrated no time dependent changes in any of the parameters examined except specimen length during the first $3 \mathrm{hrs}$ for tendons maintained in Ringer's solution. However, a decrease in apparent elastic modulus was associated with preservation by freezing.

A possible explanation for the observed shortening of fresh specimens may be found in the tendency for the tissue to absorb water when in Ringer's solution. From the data obtained, however, there seemed to be no relation of this phenomenon to any quantitative change in the stress-strain characteristics referred to initial dimensions. Thus, Smith's (1954) insistence on testing specimens within the first $\frac{1}{2}$ hr may represent an unnecessary restriction on the experimental procedure for studies on the mechanics of connective tissue. Nevertheless, some caution seems indicated; and the observed decrease in apparent elastic modulus associated with preservation by freezing brings into question the validity of the practice of pooling data from fresh and frozen specimens. 


\section{REFERENCES}

Cronkite, A. E. (1936) The tensile strength of human tendons, Anat. Rec. 64, 173-186.

Nunley. R. L. (1958) The ligamenta flava of the dog. A study of tensile and physical properties. Am. J. Phys. Med. 37.256-268.

Smith. J. W. (1954) The elastic properties of the anterior cruciate ligament of the rabbit. J. Anat. 88, 369-380.

Van Brocklin. James D. and Ellis. Donald G. (1965) A study of the mechanical behavior of toe extensor tendons under applied stress. Archs, phys. Med. 46. $369-373$.
Viidik, A. and Lewin. T. (1966) Changes in tensile strength characteristics and histology of rabbit ligaments induced by different modes of postmortal storage. Acta. orthop. scand. 37. 141-155.

Viidik, A.. Sandquist, L. and Magi. M. (1965) Influence of postmortal storage on tensile strength characteristics and histology of rabbit ligaments. Acta. orthop. scand. Suplementum 79.

Walker, L. B. Harris, Edward H.. and Benedict. James V. (1964) Stress-strain relationship in human cadaveric plantaris tendon: A preliminary study. Med. Electron. Biol. Engng. 2. 31-38. 